\title{
Enhanced autophagy is required for survival in EGFR- independent EGFR-mutant lung adenocarcinoma cells
}

\author{
Yuji Sakuma ${ }^{1,2,5}$, Shoichi Matsukuma ${ }^{1}$, Yoshiyasu Nakamura ${ }^{1}$, Mitsuyo Yoshihara ${ }^{1}$, Shiro Koizume ${ }^{1}$, Hironobu \\ Sekiguchi ${ }^{2}$, Haruhiro Saito ${ }^{3}$, Haruhiko Nakayama ${ }^{3}$, Yoichi Kameda ${ }^{4}$, Tomoyuki Yokose ${ }^{4}$, Sachiko Oguni ${ }^{5}$, Toshiro \\ $\mathrm{Niki}^{5}$ and Yohei Miyagi ${ }^{1,2}$
}

Lung cancers harboring epidermal growth factor receptor (EGFR) mutations depend on constitutive activation of the kinase for survival. Although most EGFR-mutant lung cancers are sensitive to EGFR tyrosine kinase inhibitors (TKIs) and shrink in response to treatment, acquired resistance to TKI therapy is common. We demonstrate here that two EGFRmutated lung adenocarcinoma cell lines, HCC827 and HCC4006, contain a subpopulation of cells that have undergone epithelial-to-mesenchymal transition and survive independent of activated EGFR. These EGFR-independent cancer cells, herein termed gefitinib-resistant (GR) cells, demonstrate higher levels of basal autophagy than their parental cells and thrive under hypoxic, reduced-serum conditions in vitro; this somewhat simulates the hypoxic environment common to cancerous tissues. We show that depletion of the essential autophagy gene, ATG5, by small interfering RNA (siRNA) or chloroquine, an autophagy inhibitor, markedly reduces GR cell viability under hypoxic conditions. Moreover, we show a significant elevation in caspase activity in GR cells following knockdown of ATG5. These results suggest that GR cells can evade apoptosis and survive in hostile, hypoxic environments with constant autophagic flux. We also show the presence of autophagosomes in some cancer cells from patient samples, even in untreated EGFR-mutant lung cancer tissue samples. Together, our results indicate that autophagy inhibitors alone or in combination with EGFR TKIs may be an effective approach for the treatment of EGFR-mutant lung cancers, where basal autophagy of some cancer cells is upregulated.

Laboratory Investigation (2013) 93, 1137-1146; doi:10.1038/labinvest.2013.102; published online 12 August 2013

KEYWORDS: acquired resistance; autophagy; EGFR mutation; epithelial-to-mesenchymal transition (EMT); lung adenocarcinoma

Activating mutations in the epidermal growth factor receptor (EGFR) gene are critical for the growth and survival of lung cancers. EGFR tyrosine kinase inhibitors (TKIs), such as gefitinib or erlotinib, have been shown to be effective against tumor growth during the initial stages of treatment in these cancer subtypes. However, EGFR-mutated tumors invariably acquire resistance to these EGFR TKIs, most commonly through a spontaneous secondary mutation (T790M) in $E G F R$, or via the amplification or ligand-dependent stimulation of Met kinase. ${ }^{1,2}$ Several therapeutic options to treat these resistant tumors have been developed, including WZ4002, a third-generation EGFR TKI that can effectively inhibit the kinase activity of T790M-mutated EGFR. ${ }^{3}$ In addition, combinatorial treatment with TKIs against both
EGFR and Met can induce apoptosis in Met-activated EGFRmutated cancer cells. ${ }^{4}$ EGFR-mutated tumors can also acquire resistance to EGFR TKIs through epithelial-tomesenchymal transition (EMT) ${ }^{5-8}$ Furthermore, the AXL receptor tyrosine kinase has been shown to have a key role in EMT-induced drug resistance; ${ }^{9}$ however, it remains unclear whether AXL activation invariably occurs in EGFR-mutant carcinoma cells showing an EMT phenotype.

Autophagy describes the process by which cells capture intracellular proteins, lipids, and organelles into autophagosomes, and deliver them to the lysosomes to form autophagolysosomes (also termed autolysosomes) for degradation. These degradation products are then exported from autophagolysosomes into the cytoplasm for recycling.

\footnotetext{
${ }^{1}$ Molecular Pathology and Genetics Division, Kanagawa Cancer Center Research Institute, Yokohama, Japan; ${ }^{2}$ Laboratory for Molecular Diagnostics, Kanagawa Cancer Center Hospital, Yokohama, Japan; ${ }^{3}$ Department of Thoracic Oncology, Kanagawa Cancer Center Hospital, Yokohama, Japan; ${ }^{4}$ Department of Pathology, Kanagawa Cancer Center Hospital, Yokohama, Japan and ${ }^{5}$ Department of Pathology, Jichi Medical University, Tochigi, Japan Correspondence: Dr Y Sakuma, MD, PhD, Department of Pathology, Jichi Medical University, 3311-1 Yakushiji, Shimotsuke, Tochigi 329-0498, Japan. E-mail: ysakuma@jichi.ac.jp 
This intracellular recycling function maintains both normal cells and cancer cells in a healthy state, and thereby promotes the survival of malignant cells once the tumor is established. The core molecular machinery required for the formation of an autophagosome comprises a heterogeneous group of proteins encoded by autophagy-related $A T G$ genes, such as ATG5, Beclin1 (also known as ATG6), and microtubule-associated protein 1 light chain 3 (LC3), a human homolog of yeast ATG8. ${ }^{10-12}$ The conversion of cytosolic LC3-I to autophagosome-bound LC3-II acts as a biochemical marker of active autophagy, ${ }^{10-16}$ and it has been increasingly appreciated that the immunohistochemical detection of cytoplasmic LC3 puncta in formalin-fixed, paraffinembedded specimens (FFPE) correlates with the amount of autophagosomes or autophagolysosomes. ${ }^{14,17,18}$ Although it is known that cancer cells with Ras mutations are addicted to upregulated autophagic flux, ${ }^{10,11,13,14}$ little has been done to clarify the role of autophagy in EGFR-driven carcinoma cells. ${ }^{19,20}$

In this study, we demonstrate that gefitinib-resistant (GR) EGFR-mutant lung carcinoma cells with an EMT phenotype can survive under hypoxic conditions in vitro in the absence of EGFR T790M mutation or Met, EGFR, or AXL activity because of enhanced autophagic flux. Furthermore, using immunohistochemistry and electron microscopy, we also demonstrate cytoplasmic, granular expression of LC3A (an isoform of LC3) in untreated EGFR-mutant lung cancer tissues, as well as the formation of autophagosomes in these cells.

\section{MATERIALS AND METHODS}

The experimental procedures were approved by the Institutional Review Board at the Kanagawa Cancer Center, Japan.

\section{Cell Culture and Drugs}

Two EGFR-mutant lung adenocarcinoma cell lines, HCC827 (del E746-A750) and HCC4006 (del L747-E749 + A750P), were purchased from the American Type Culture Collection (Manassas, VA, USA) and maintained in RPMI1640 media containing $10 \%$ fetal calf serum (FCS) and antibiotics at $37^{\circ} \mathrm{C}$ in a humidified incubator with $20 \% \mathrm{O}_{2}+5 \% \mathrm{CO}_{2}$. A cancer tissue microenvironment was simulated with RPMI1640 media containing $1 \%$ FCS and antibiotics at $37^{\circ} \mathrm{C}$ in a humidified incubator with $1 \% \mathrm{O}_{2}+5 \% \mathrm{CO}_{2}$. Two EGFR TKIs, gefitinib (Biaffin GmbH \& Co KG, Kassel, Germany) and WZ4002 (Selleck Chemicals, Houston, TX, USA), were used in this study, as well as an autophagy-inducing drug, salinomycin (Sigma-Aldrich, St Louis, MO, USA) ${ }^{21}$ and a late-stage inhibitor of autophagy, chloroquine (CQ) diphosphate salt (Sigma-Aldrich). ${ }^{11}$

\section{Generation of GR HCC827 and HCC4006 Cells}

HCC827 and HCC4006 cells were cultured in the presence of $1 \mu \mathrm{M}$ gefitinib until they acquired resistance. After surviving 20 passages in the presence of the drug, single-cell cloning was performed using cloning cylinders. GR subclones were successfully expanded in media containing $1 \mu \mathrm{M}$ gefitinib and were termed HCC827 GR1-5 or HCC4006 GR1-3 cells, respectively. HCC827 GR2 and HCC4006 GR3 sublines are characterized in detail in this study.

\section{Loop-hybrid Mobility Shift Assay}

A loop-hybrid mobility shift assay (LH-MSA) was used for the detection of T790M mutation in EGFR. ${ }^{22-24}$ Briefly, cell-derived DNA was subjected to PCR with a primer pair (5'-ctccaccgtgcaagctcatca- $3^{\prime}$ and $5^{\prime}$-gtactgggagccaatattgtctttg$\left.3^{\prime}\right)$ and then a Cy5-labeled LH probe was added to the product at a final concentration of $240 \mathrm{nM}$ (Cy5-5'ctccaccgtgcaagctcatcacATATATATAgcagctcatgcccttcggctgcc- $3^{\prime}$; insertion is denoted by capital letters) using a hybridization procedure of $94{ }^{\circ} \mathrm{C}$ for $2 \mathrm{~min}, 55^{\circ} \mathrm{C}$ for $45 \mathrm{~s}$, and $68^{\circ} \mathrm{C}$ for $4 \mathrm{~min}$. The reacted products were separated by $10 \%$ polyacrylamide gel electrophoresis (C10L; Atto Inc, Tokyo, Japan) and detected with STORM860 (GE Healthcare, Buckinghamshire, UK) for Cy5 fluorescence (at $635 \mathrm{~nm}$ with an LP650 filter).

\section{RNA Interference Assay}

Cells $\left(20 \times 10^{5}\right)$ were plated in 94-mm culture dishes, grown for $24 \mathrm{~h}$, and then transfected with siRNA duplexes targeting EGFR (SI02663983), AXL (SI00605311), or ATG5 (SI02655310), and with negative control siRNA duplexes (1027281) (Qiagen, Valencia, CA, USA) using Lipofectamine RNAiMAX Reagent and OPTI-MEM I (Invitrogen), according to the manufacturer's recommendations.

\section{Quantitative RT-PCR}

Total RNA was extracted with the RNeasy Mini Kit (Qiagen) and $1 \mu \mathrm{g}$ RNA was reverse transcribed into cDNA using the QuantiTect Reverse Transcription Kit (Qiagen). Real-time PCR was performed using the QuantiTect Primer Assay and QuantiFast SYBR Green PCR Kit (Qiagen) in a LightCycler 480 (Roche Applied Science, Mannheim, Germany). $\beta$-actin mRNA (Hs_ACTB_2_SG) was used to standardize the quantity of LC3A mRNA (Hs_MAP1LC3A_1_SG). The relative expression of LC3A mRNA between parental cells and GR cells was calculated by the difference in the threshold cycle (comparative $\mathrm{C}_{\mathrm{T}}$ method). Data are presented as the mean \pm s.d. of triplicate experiments.

\section{Western Blotting}

Western blotting for the expression of poly(ADP-ribose) polymerase-1 (PARP-1), total EGFR, phosphorylated EGFR (pEGFR), $\gamma \mathrm{H} 2 \mathrm{AX}$, and $\beta$-actin was performed as described previously. ${ }^{25,26}$ Additional primary antibodies for immunoblotting were anti-E-cadherin (34; 1:500 dilution; BD Biosciences, Franklin Lakes, NJ, USA), anti-Ep-CAM (C-10; 1:1000 dilution; Santa Cruz Biotechnology, Santa Cruz, CA, USA), anti-Vimentin (SP20; 1:2000 dilution; Epitomics, Burlingame, CA, USA), anti-Met (C-12; 1:2000 
dilution; Santa Cruz Biotechnology), anti-CD44 (EPR1031Y; 1:2000 dilution; Epitomics), anti-AXL (C44G1; 1:1000 dilution; Cell Signaling, Tokyo, Japan), anti-LC3A (D50G8; 1:1000 dilution; Cell Signaling), anti-ATG5 (D5G3; 1:1000 dilution; Cell Signaling), and anti-Beclin1 (D40C5; 1:1000 dilution; Cell Signaling). Band intensity levels on X-ray films were normalized to $\beta$-actin using Image $\mathrm{J}$ software $(\mathrm{NIH}$, Bethesda, MD, USA). Each experiment was performed in triplicate.

\section{Assessment of Cell Viability and Apoptosis}

Cell viability was assessed by trypan blue exclusion or using a WST-1 assay (Roche Applied Science). Apoptosis was assessed by western blot analysis of the cleaved PARP-1 or Caspase-Glo 3/7 Assay (Promega, Madison, WI, USA), as previously described. ${ }^{25,26}$

\section{Primary EGFR-Mutant Lung Cancer Tissues}

Tumor samples were obtained from 20 EGFR-mutant lung cancer patients who underwent surgery at the Kanagawa Cancer Center Hospital between August 2002 and August 2007. The patients included 10 men and 10 women, with a median age of 58.5 years (range 38-80 years). Aside from one patient (adenosquamous cell carcinoma), all tumors were classified as adenocarcinoma by histology. EGFR mutations were identified as a deletion mutation in exon $19(n=11)$ or an L858R point mutation in exon $21 \quad(n=9)$. None of the patients received neoadjuvant chemotherapy before surgery.

\section{Immunohistochemistry}

Immunohistochemical staining for LC3A was performed on FFPE tissue sections of lung cancers. Tissue sections were retrieved by autoclave treatment $\left(121^{\circ} \mathrm{C}\right.$ for $\left.15 \mathrm{~min}\right)$ in a citrate buffer ( $\mathrm{pH}$ 6.0). Slides were then incubated overnight at $4{ }^{\circ} \mathrm{C}$ with anti-LC3A (1:3200 dilution). Immunohistochemical staining of peripheral nerve bundles in the tissue was used as an internal positive control. ${ }^{14,17}$ The cancer tissue was judged as positive for LC3A expression when 5\% or more carcinoma cells stained positive for LC3A.

\section{Transmission Electron Microscopy}

Ultrastructural studies were performed using an FFPE tissue sample obtained from one patient whose tumor showed highest positivity for LC3A expression by immunohistochemistry. The tissue was deparaffinized and then refixed in $2.5 \%$ glutaraldehyde overnight at $4{ }^{\circ} \mathrm{C}$. After post fixation in $1 \%$ osmium tetroxide for $1 \mathrm{~h}$ and dehydration, the sample was embedded in epon. Conventional thin sections were collected on uncoated grids, stained with uranyl acetate and lead citrate, and then examined with a JEM-1400Plus transmission electron microscope (JEOL, Tokyo, Japan).

\section{Statistical Analysis}

Differences in caspase activity or cell viability between untreated and treated cells were evaluated by paired $t$-tests. Differences in the expression levels of total EGFR or pEGFR, expression levels of LC3A mRNA, and cell viability between parental cells and GR cells were evaluated by unpaired $t$-tests. $P$-values $<0.05$ were considered significant. All statistical calculations were performed with the JMP software (JMP for Windows version 7; SAS Institute Japan; Tokyo, Japan).

\section{RESULTS}

\section{GR Cells Display an EMT Phenotype in the Absence of EGFR T790M Mutation or Met Activation}

We first confirmed that HCC827 GR2 and HCC4006 GR3 cells had the same deletion mutation in EGFR as their respective parental cells, and that neither GR cell line carried the T790M mutation by LH-MSA and sequencing (Figure 1a and data not shown). In addition, Met expression in GR cells was considerably lower than that in the parental cells (Figure 1b). Combinatorial therapy with gefitinib and PHA665752, a Met TKI, did not induce apoptosis in GR cells (data not shown), indicating that these GR cells do not require Met activation for survival. These findings indicate that both HCC827 GR2 and HCC4006 GR3 cells lack the two recognized molecular mechanisms that confer resistance to EGFR TKI therapy.

Following this, we used western blot analysis to show that two epithelial markers, E-cadherin and Ep-CAM, were markedly downregulated in GR cells as compared with the parental cells, and that the mesenchymal marker vimentin was substantially elevated in GR cells (Figure 1b). Using light microscopy, we observed that GR cells were spindle shaped but the parental cells had a cobblestone-like appearance (Figure 1c and data not shown). These findings provided evidence for the EMT phenotype of GR cells. We also observed a marked increase in CD44 expression in GR cells as compared with their parental cells (Figure 1b). As parental HCC827 and HCC4006 cell lines contain a minor subpopulation of cells that express CD44 $(<1 \%),{ }^{5}$ it is likely that gefitinib treatment selected for these pre-existing GR cells from the parental cell culture.

Interestingly, in HCC827 GR2 cells, we observed a marked decrease in the expression of pEGFR relative to the parental cells, but no change in total EGFR expression (Figure 1d). By comparison, although pEGFR was also significantly reduced in HCC4006 GR3 cells as compared with parental cells, there was a similar significant reduction in total EGFR levels in these cells (Figure 1e). This marked decrease in EGFR kinase activity suggests that GR cells are less dependent on EGFR signaling for survival than the parental cells.

GR Cells do not Depend on EGFR Signaling for Survival To confirm the hypothesis that GR cells are not dependent on EGFR signaling, we sought to examine changes in the expression of several cell death markers between GR and their 
a

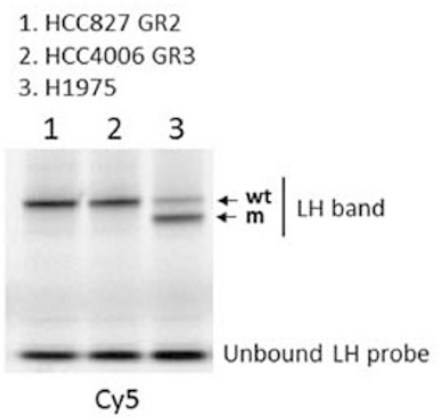

b

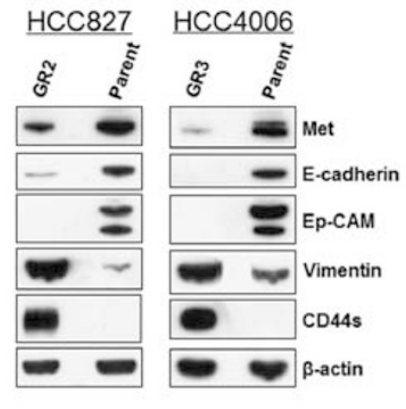

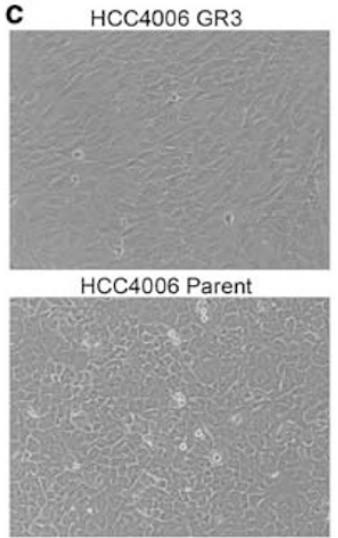

d

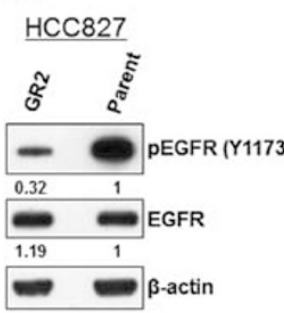

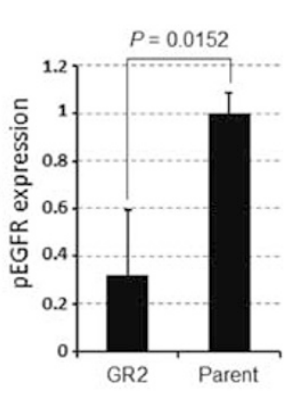

e

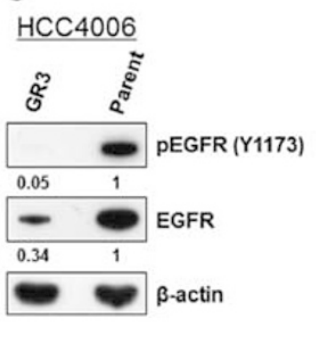

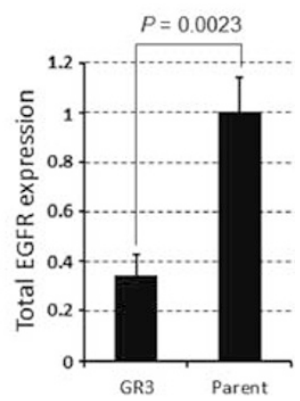

Figure 1 Gefitinib-resistant (GR) cells displayed an epithelial-to-mesenchymal transitioned (EMT) phenotype. (a) Detection of epidermal growth factor receptor (EGFR) T790M mutation by the loop-hybrid mobility shift assay (LH-MSA). Representative heterozygous LH band patterns of the wild-type (wt) and the T790M mutation (m) after LH-MSA using an LH probe are illustrated. The mutation is present in H1975 cells (an EGFR-mutant cell line having the T790M mutation), but not HCC827 GR2 or HCC4006 GR3 cells. (b) Western blots of HCC827 or HCC4006 GR and their respective parental cells. CD44s, standard isoform of CD44. (c) Representative phase-contrast images of HCC4006 GR3 cells and parental cells. (d, e) (Left panel) Western blots of (d) HCC827 GR2 and parental cells and (e) HCC4006 GR3 and parental cells. The expression levels of phosphorylated EGFR (pEGFR) and total EGFR were normalized to $\beta$-actin. (Right panel) Quantification of (d) pEGFR expression and (e) total EGFR expression. Results are expressed as the mean ( $n=3$ ) and s.d.

parental cells after EGFR TKI treatment. In parental HCC827 and HCC4006 cells, EGFR autophosphorylation was suppressed with gefitinib or WZ4002, and the cells clearly underwent apoptosis, with an increased expression of $\gamma \mathrm{H} 2 \mathrm{AX}$ (a DNA damage marker), a significant elevation in caspase 3/ 7 activity, and cleavage of PARP-1 (Figure 2a and b). However, these markers remained unchanged in GR cells in the presence of EGFR TKIs (Figures $2 \mathrm{a}$ and $\mathrm{b}$ ). In addition, siRNA-mediated EGFR knockdown also did not induce apoptosis in both GR cell lines (Figure 2c). These results confirmed our hypothesis that GR cells do not depend on EGFR signaling for survival, despite the presence of the same activating mutations in EGFR as their parental cells. Both gefitinib and WZ4002 almost completely suppressed EGFR autophosphorylation in HCC827 GR2 cells, as well as its parental cells (Figure 2a). Further, total EGFR was upregulated in HCC4006 GR3 cells following treatment with EGFR TKIs (Figure 2a), suggesting that activated drug efflux was not responsible for the GR cells' resistance to EGFR TKIs. These responses to gefitinib treatment observed in both GR cells also support the absence of EGFR T790M mutation in GR cells, because gefitinib cannot suppress the kinase activity nor affect the expression levels of mutant EGFR with the T790M mutation. ${ }^{2,3}$

\section{AXL Kinase does not have a Critical Role in GR Cell Survival}

Because AXL kinase has been shown to have a key role in EMT-induced drug resistance, we sought to rule out its involvement in GR cells. HCC4006 GR3 cells showed a higher expression of AXL kinase than the parental cells. Comparatively, AXL expression in HCC827 GR2 cells was too low for detection, with only faint protein expression observed in the parental cells (Figure 3a). In HCC4006 GR3 cells, siRNAmediated AXL knockdown followed by gefitinib treatment had no effect on $\gamma \mathrm{H} 2 \mathrm{AX}$ or PARP-1 cleavage (Figure $3 \mathrm{~b}$ ). As expected, HCC827 GR2 cells were insensitive to the same treatment (data not shown). These results indicate that GR cells do not require AXL kinase activity for survival.

\section{GR Cells have Higher Levels of Basal Autophagy than Parental Cells}

Next, we sought to test the role of autophagy in GR cells using autophagosome-bound LC3A-II as a biochemical 
a

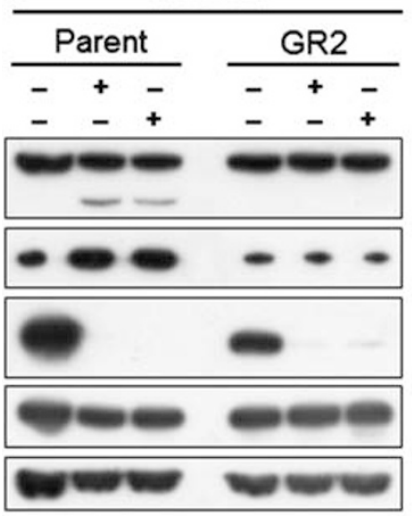

b

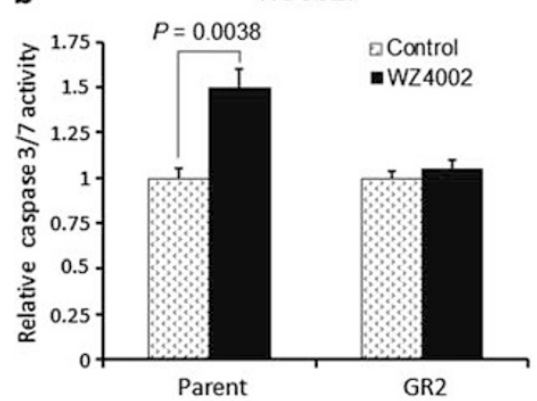

Parent

\section{HCC4006}

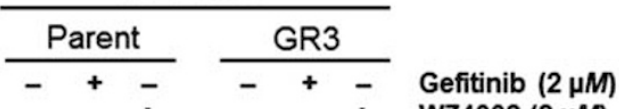

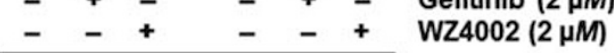
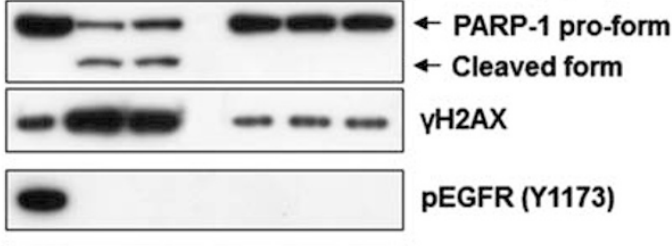

pEGFR (Y1173)

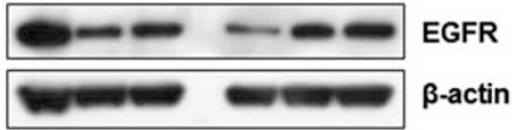

HCC4006

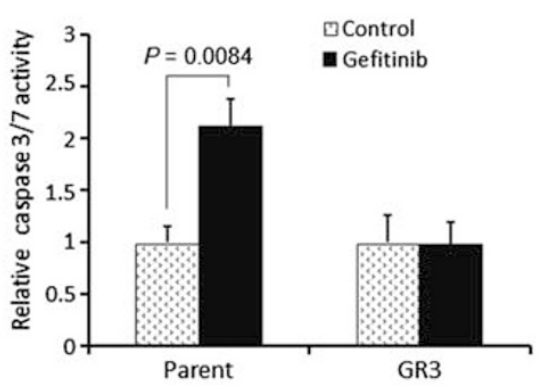

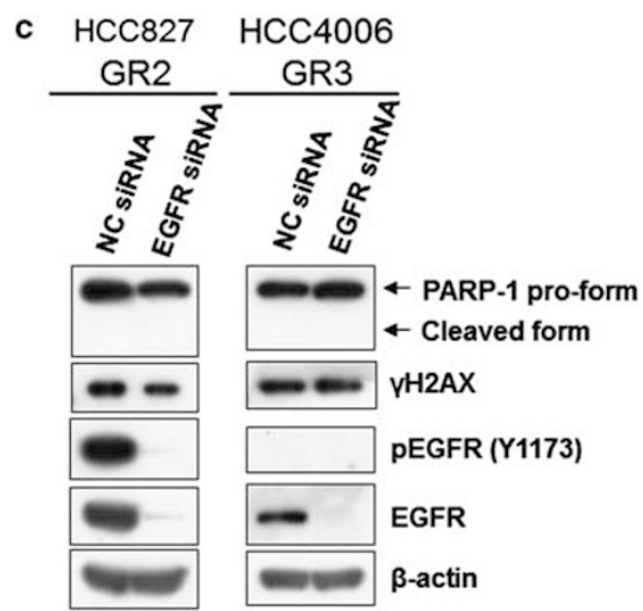

Figure 2 Gefitinib-resistant (GR) cells do not depend on epidermal growth factor receptor (EGFR) activity for survival. (a) Western blots examining the effects of gefitinib or WZ4002 treatment on apoptosis in parental cells and GR cells. Cells were untreated or treated with the indicated drug for $48 \mathrm{~h}$. (b) Caspase $3 / 7$ activity in HCC827 and HCC4006 cells. Cells were untreated or treated with the indicated drug ( $2 \mu \mathrm{M})$ for $24 \mathrm{~h}$. Caspase activity was normalized to an arbitrary unit of 1.0 for the mean of three untreated wells. Results are expressed as the mean $(n=3)$ and s.d. (c) Western blots examining the effects of EGFR depletion on apoptosis in HCC827 GR2 and HCC4006 GR3 cells. Cells were transfected with negative control (NC) small interfering RNA (siRNA) or EGFR siRNA, and cultured for $72 \mathrm{~h}$.

marker of active autophagy. GR cells cultured in $20 \%$ oxygen and 10\% FCS (hereafter called 'normoxic conditions') had a much higher expression of LC3A mRNA and autophagosome-bound LC3A-II protein as compared with those levels in the parental cells (Figures $4 \mathrm{a}-\mathrm{c}$ ). This was true even when GR and their parental cells were treated by salinomycin, an autophagy-inducing drug, ${ }^{21}$ or $\mathrm{CQ}$, a late-stage inhibitor of autophagy. ${ }^{11}$ These findings indicate that GR cells have significantly higher levels of basal autophagy than their respective parental cells. Although both parental cells were able to grow in the presence of CQ, HCC827 GR2 cells treated with CQ showed minimal proliferation, and HCC4006 GR3 cell viability was decreased (Figure 4d). These results suggest that GR cells are more dependent on autophagic flux for survival or proliferation than their parental cells, even under normoxic conditions. GR cells showed 


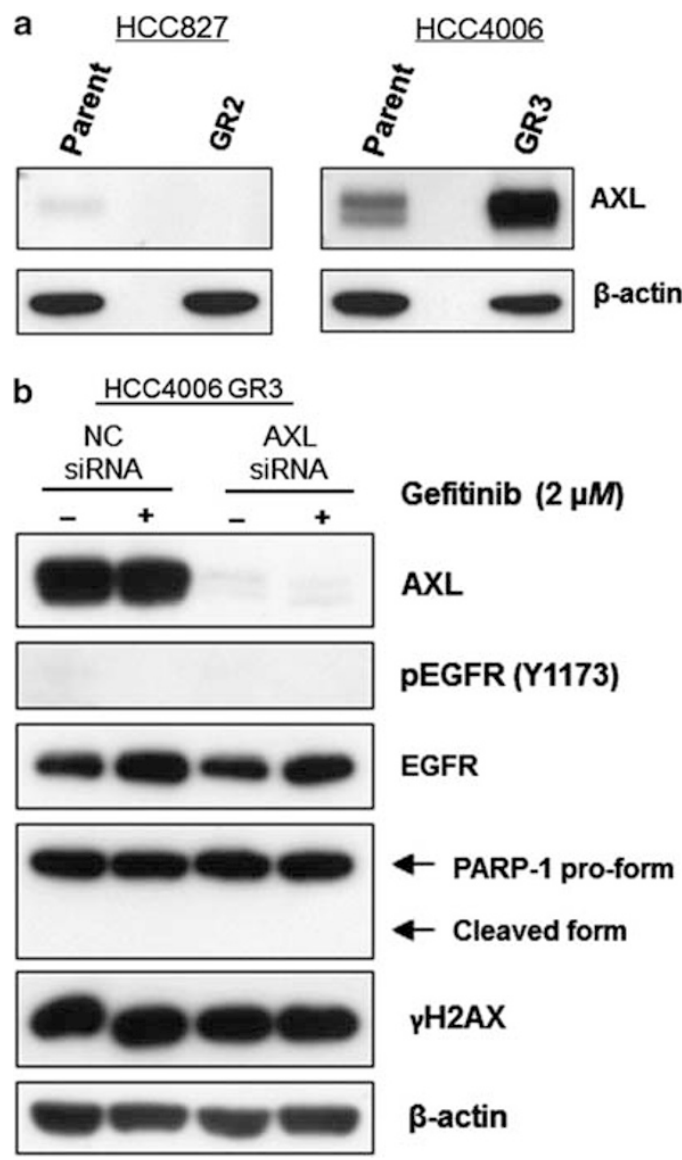

Figure 3 Gefitinib-resistant (GR) cells can survive independently of AXL receptor tyrosine kinase. (a) Western blots of HCC827 or HCC4006 parental and GR cells for the AXL kinase. (b) Western blots examining the effects of AXL depletion, gefitinib treatment, or a combination of $A X L$ depletion and gefitinib or neither in HCC4006 GR3 cells. Cells transfected with negative control (NC) small interfering RNA (siRNA; $10 \mathrm{nM}$ ) or AXLspecific siRNA $(10 \mathrm{nM})$ were cultured for $72 \mathrm{~h}$ and then untreated or treated with gefitinib $(2 \mu \mathrm{M})$ for another $48 \mathrm{~h}$.

slightly decreased expression of ATG5 as compared with their parental cells, and both GR cells and parental cells expressed Beclin1 in almost equal amounts (Figures $4 \mathrm{~b}$ and c).

\section{High Basal Autophagy Promotes Survival of GR Cells Under Hypoxia}

Autophagy in cancer cells and EMT are both known to be induced in hypoxic microenvironments, ${ }^{10,11,27-31}$ with oxygen concentrations in lung cancer tissues shown to be as low as $\sim 1 \% .{ }^{29}$ Although GR cells analyzed in this study were established under normoxia, we hypothesized that their high basal autophagy would facilitate their survival under $1 \%$ oxygen and 1\% FCS (hereafter referred to as hypoxic conditions'). Figure 5a shows that HCC827 GR2 and HCC4006 GR3 cells proliferated under hypoxic conditions, whereas their respective parental cells showed reduced viability in the hostile environment. To test the hypothesis mentioned above, we depleted ATG5 expression using siRNA and found that ATG5 depletion significantly reduced GR cell viability under hypoxic conditions (Figure $5 b$ ). This marked decline in viability with ATG5 depletion was probably due to impaired autophagic flux, as confirmed by a decrease in the expression of the LC3A-II form in the ATG5-depleted GR cells (Figure $5 \mathrm{c}$ ). We also observed significantly enhanced caspase activity in ATG5-depleted GR cells compared with control cells (Figure 5d), suggesting that GR cells, at least in part, underwent apoptosis (Figures $5 \mathrm{~b}$ and e). Beclin1 depletion caused a similar decrease in GR cell viability (data not shown) and CQ treatment dose-dependently decreased GR cell viability under hypoxic conditions (Figure 5f). Together, these results indicate that GR cells can thrive and proliferate under hypoxic conditions because of their high basal autophagy.

\section{Autophagosomes are Formed in Untreated EGFR- Mutant Lung Cancer Tissues}

To confirm the presence of autophagosomes in tissue samples, we collected tumor samples from 20 EGFR-mutant lung cancer patients, with all but one patient classified as adenocarcinoma by histology, and performed immunohistochemistry for LC3A protein using FFPE sections. We found that 14 out of the 20 EGFR-mutant lung carcinoma tissues were positive for LC3A by immunohistochemistry, ranging from 5 to $60 \%$ positivity (median, 10\%). LC3A protein localized to the cytoplasm of carcinoma cells with a granular staining pattern, which is highly suggestive of autophago(lyso)somes (Figure 6a). ${ }^{14,17,18}$ To confirm whether the cytoplasmic distribution of LC3A protein in carcinoma cells reflects autophagosome formation, an LC3A-positive adenocarcinoma specimen was examined with transmission electron microscopy. We found that carcinoma cells contained vesicles with a double membrane-like structure that engulfed the cytoplasm, which is characteristic of autophagosomes (Figure 6b). ${ }^{14,17}$ LC3A-positive cells were not detected in non-tumoral tissues, except for in bronchial (or bronchiolar) epithelial cells and peripheral nerve fibers.

\section{DISCUSSION}

We have shown that two EGFR-mutant lung adenocarcinoma cell lines, HCC827 and HCC4006, have subpopulations of GR cells that display an EMT phenotype and can survive independent of EGFR signaling. This observation that EGFRindependent cells are inherent to EGFR-mutant cell lines could be one reason why EGFR TKI therapy cannot permanently eradicate EGFR-mutant lung cancers in the clinic. Although the GR cells analyzed here were selected from cell lines in vitro, studies show that some EGFR-mutant lung cancers with acquired TKI resistance display an EMT phenotype in the clinic. ${ }^{5-8}$ Like EGFR-mutant lung cancer, chronic myeloid leukemia, which has the Bcr-Abl fusion gene, ${ }^{32}$ and gastrointestinal stromal tumors, which harbors an activating mutation in the c-kit gene, ${ }^{33}$ are addicted to constitutively activated tyrosine kinases. Intriguingly, these 
a

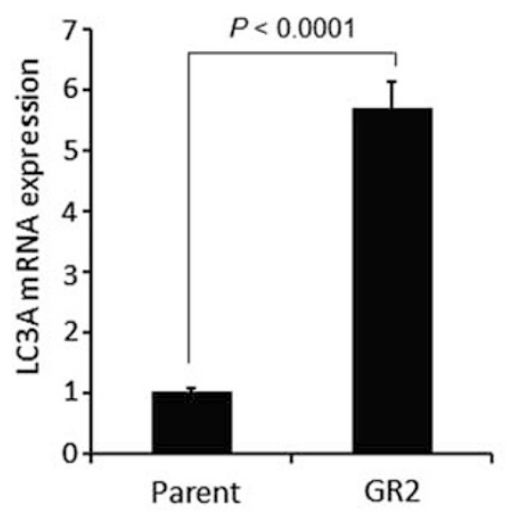

b

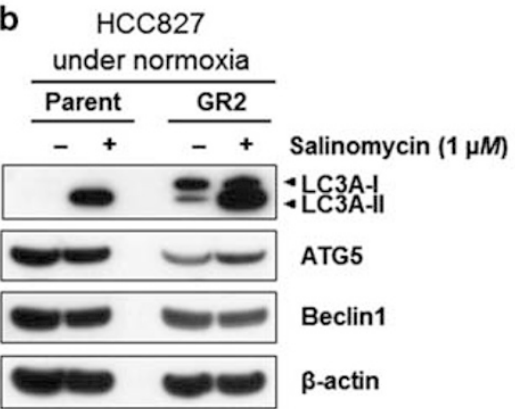

d

HCC827

upon chloroquine

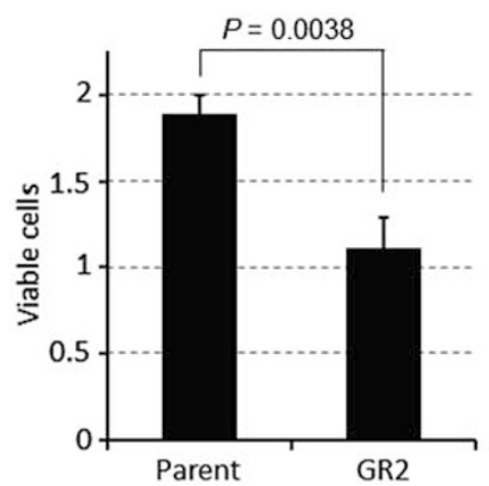

HCC4006 under normoxia

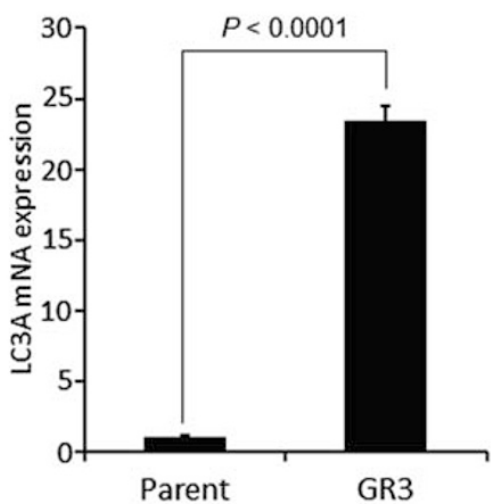

C

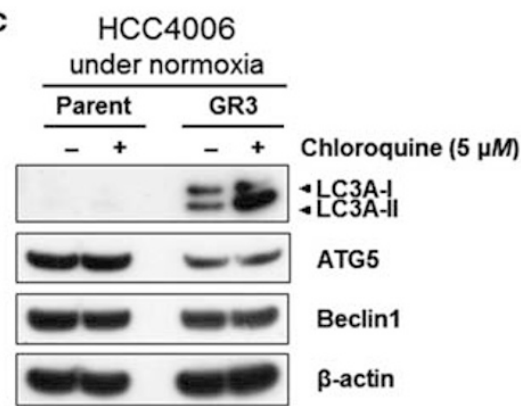

HCC4006

upon chloroquine

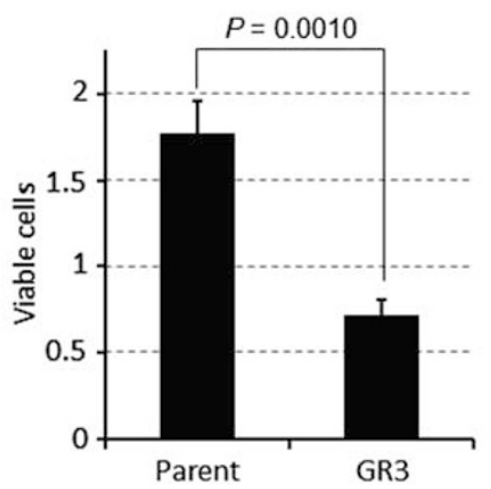

Figure 4 Gefitinib-resistant (GR) cells have higher levels of basal autophagy. (a) Real-time RT-PCR for the expression of LC3A mRNA in parental cells and GR cells from HCC827 or HCC4006 cells. (b) Western blots examining the effects of salinomycin (1 $\mu \mathrm{M})$ for $48 \mathrm{~h}$ in HCC827 parental and GR2 cells. (c) Western blots examining the effects of chloroquine (CQ; $5 \mu \mathrm{M})$ for $48 \mathrm{~h}$ in HCC4006 parental and GR3 cells. (d) Viability of HCC827 or HCC4006 parental and GR cells following CQ treatment. Cells were treated with CQ $(15 \mu \mathrm{M})$ for $72 \mathrm{~h}$ under normoxic conditions $(20 \%$ oxygen and $10 \% \mathrm{FCS})$. Cell viability was assessed by trypan blue exclusion and the results were normalized to an arbitrary unit of 1.0 for viable cell number immediately before treatment. Results are expressed as the mean $(n=3)$ and s.d.

two tumors include a subset of cells that do not require the activated kinase for survival, ${ }^{32,33}$ similar to the EGFR-mutant GR cells in our study.

In this study, we have also uncovered that GR cells have a higher degree of basal autophagy than their parental cells, which endows them with the potential to evade apoptosis and proliferate even under hypoxic conditions in vitro. These hypoxic conditions in vitro are reminiscent of the hostile environment within human cancer tissues. ${ }^{29-31}$ Autophagy confers cytoprotection to cancer cells through the collection, degradation, and recycling of intracellular material in response to stress. ${ }^{10-14}$ Here, we show that genetic and pharmacological inhibition of autophagy results in a marked decrease in GR cell viability under hypoxic conditions, and 
a

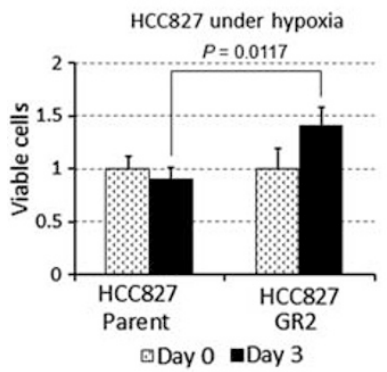

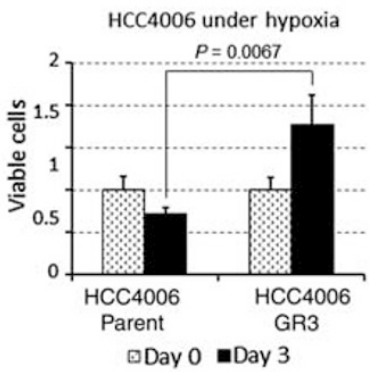
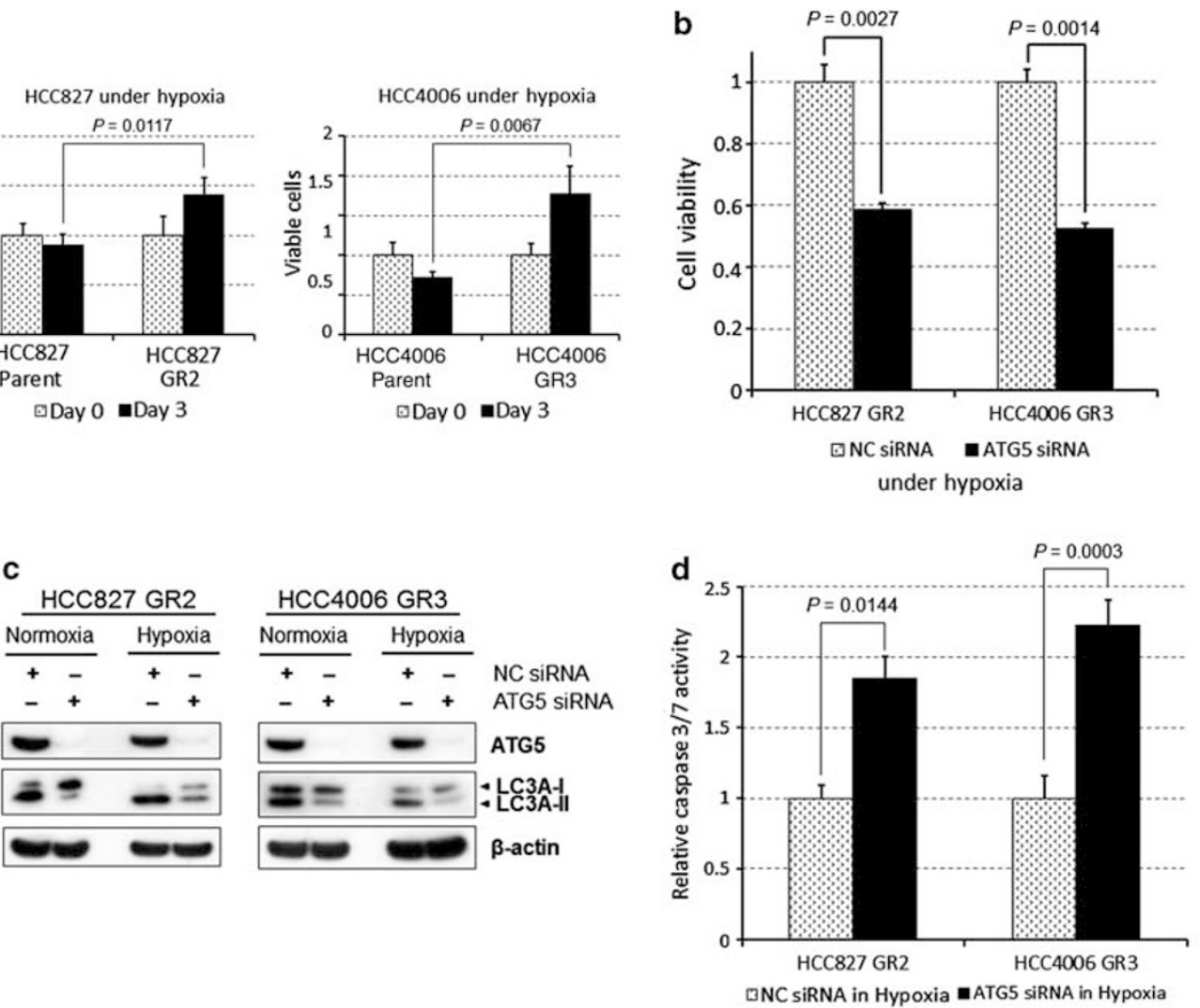

e CC827 GR2 under hypoxia
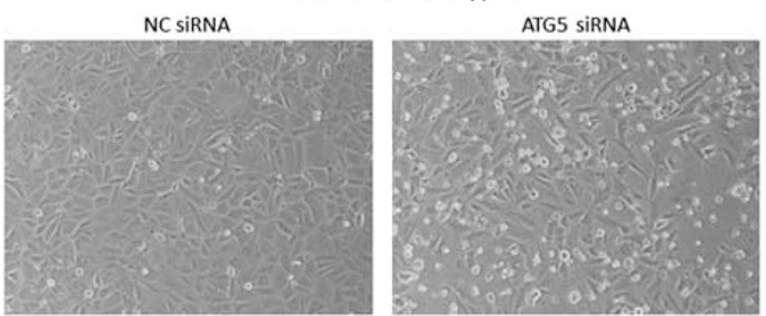

Figure 5 High basal autophagy promotes gefitinib-resistant (GR) cell survival under hypoxia. (a) Viability of HCC827 or HCC4006 parental and GR cells under hypoxic conditions. Cells were grown under normoxic conditions ( $20 \%$ oxygen and $10 \% \mathrm{FCS})$ for $24 \mathrm{~h}$, and then cultured under hypoxic conditions ( $1 \%$ oxygen and $1 \% \mathrm{FCS}$ ) for another $72 \mathrm{~h}$. Cell viability was assessed by trypan blue exclusion before hypoxic conditions (day 0 ) and $72 \mathrm{~h}$ after hypoxia induction (day 3). (b) HCC827 GR2 and HCC4006 GR3 cells were transfected with negative control (NC) small interfering RNA (siRNA; $10 \mathrm{nM}$ ) or ATG5 siRNA $(10 \mathrm{nM})$, cultured under normoxic conditions for $24 \mathrm{~h}$, and then grown under hypoxic conditions for another $72 \mathrm{~h}$. Viability was determined using a WST-1 assay. (c) Western blots examining the effects of ATG5 depletion in HCC827 GR2 and HCC4006 GR3 cells. Cells were transfected with NC siRNA $(10 \mathrm{nM})$ or ATG5 siRNA $(10 \mathrm{nM})$, cultured under normoxic conditions for $24 \mathrm{~h}$, and then grown under normoxic or hypoxic conditions for another $72 \mathrm{~h}$. (d) Caspase 3/7 activity in HCC827 GR2 and HCC4006 GR3 cells under hypoxia. Cells transfected with NC siRNA or ATG5 siRNA were seeded at initial density of $0.5 \times 10^{5}$ cells/well and grown under normoxic conditions for $24 \mathrm{~h}$, followed by hypoxic conditions for $48 \mathrm{~h}$. Caspase activity was normalized to an arbitrary unit of 1.0 for the mean of three NC siRNA-transfected wells. Columns, mean ( $n=3$ ); bars, s.d. (e) Representative phase-contrast images of HCC827 GR2 cells transfected with NC siRNA or ATG5 siRNA under hypoxia, cultured as in panel b. (f) HCC827 GR2 and HCC4006 GR3 viability after chloroquine (CQ) treatment under hypoxic conditions. Cells were seeded and grown under normoxic conditions for $24 \mathrm{~h}$ and then untreated or treated with CQ $(10 \mu \mathrm{M}$ or $20 \mu \mathrm{M})$ under hypoxia for another $72 \mathrm{~h}$. Viability was determined using a WST-1 assay. $* * P<0.01$.

that ATG5 depletion under hypoxic conditions also causes GR cells to, at least in part, undergo apoptosis. These findings are consistent with previous reports showing that the inhibition of autophagic flux in Ras-mutant cancer cells results in mitochondrial dysfunction, an accumulation of cytotoxic reactive oxygen species, and increased DNA 
a

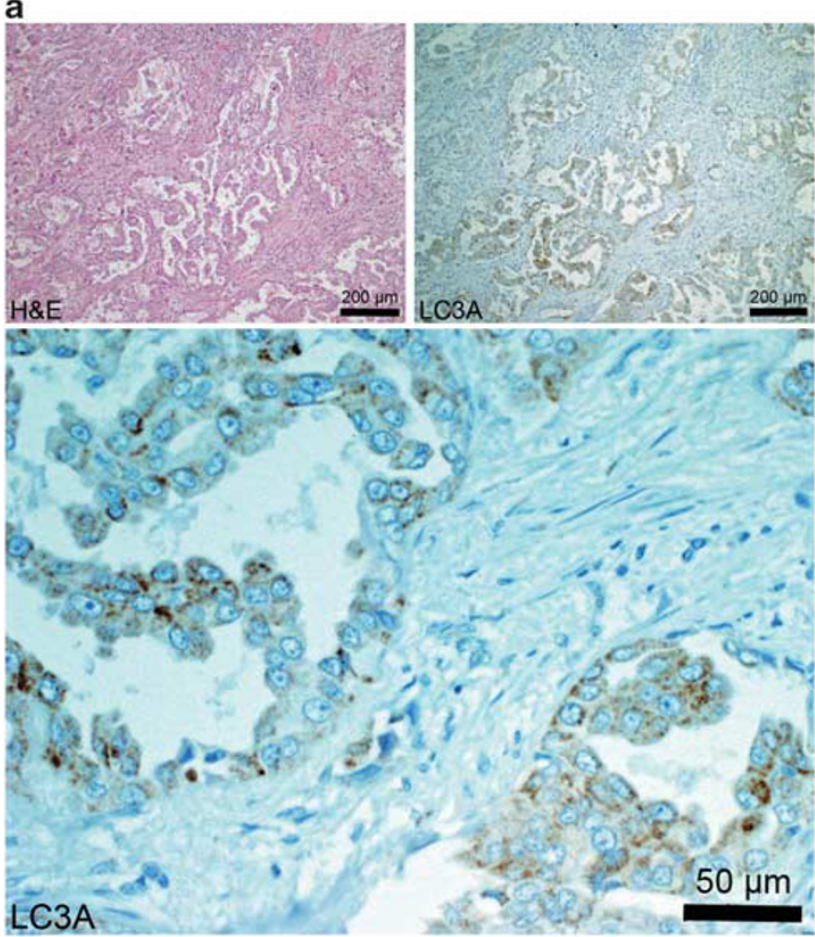

b

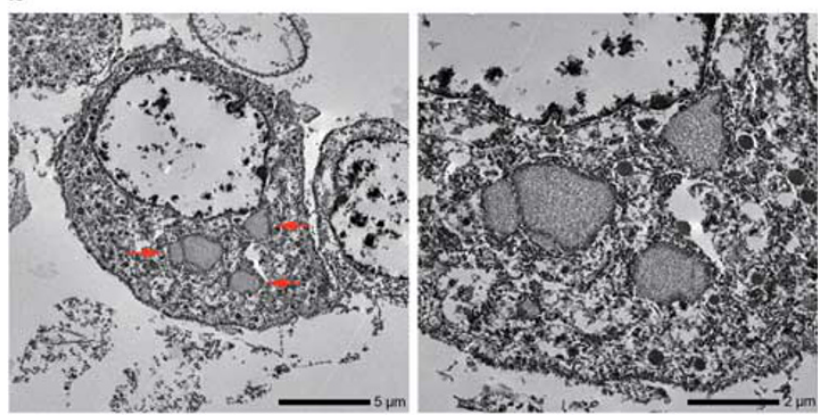

Figure 6. Autophagosomes are formed in carcinoma cells in epidermal growth factor receptor (EGFR)-mutant lung cancer tissues. (a) An untreated EGFR-mutant lung adenocarcinoma tissue expressing LC3A protein. (Upper left) Hematoxylin-eosin staining. (Upper right) Immunohistochemical staining for LC3A. (Lower) High-powered magnification of an LC3A-positive cancer tissue showing cytoplasmic, granular staining. (b) Electron microscopic analysis of the same tissue presented in panel a. (Left) Vesicles with a double membrane-like structure are indicated by arrows in red. (Right) High-powered magnification of the vesicles.

damage. ${ }^{13,14}$ Autophagy has been recently reported to have a key role in the acquired resistance to erlotinib in PC9 cells, another EGFR-mutant lung adenocarcinoma cell line. ${ }^{19}$ Furthermore, one report shows that erlotinib induces autophagy as well as apoptosis in parental HCC827 and HCC4006 cells, and that inhibiting autophagy significantly enhances sensitivity to erlotinib in both cell lines. ${ }^{20}$ These findings are analogous to those already reported in chronic myeloid leukemia cells, where suppression of autophagy can potentiate apoptotic cell death induced by imatinib, a potent Abl TKI. ${ }^{34}$ Together with our findings, these reports suggest that autophagy inhibitors could be effective not only in inducing apoptosis in EGFR TKI-resistant cells, but also in enhancing cellular susceptibility to EGFR TKIs in TKIsensitive cells.

Most of the EGFR-mutant lung cancer tissues examined in our study included $5 \%$ or more carcinoma cells with cytoplasmic, granular staining of LC3A. This pattern of LC3A staining is highly suggestive of autophagosomes, and our electron microscopy results support autophagosome formation in the cytoplasm of some cancer cells. ${ }^{14,17,18}$ However, the number of LC3A-positive carcinoma cells seems to be relatively low (median, 10\%). This is probably because none of the tumor tissues examined had received neoadjuvant therapy, including EGFR TKIs, and thus the tumor tissues correspond more to the 'parental cells' in our in vitro experiments. All of the lung cancer tissues stained for LC3A retained an epithelial phenotype and did not involve carcinoma cells with mesenchymal-like features as seen in our GR cell culture. Hence, it remains to be elucidated to what extent autophagy is activated in EGFR-mutant lung cancer tissue samples that have acquired resistance to EGFR TKIs and undergone EMT.

We could not confirm previous findings that EGFR-mutant lung cancer cells with an EMT phenotype are dependent on AXL and EGFR for survival. ${ }^{9}$ In HCC827 GR2 cells, AXL kinase expression was too low for detection by western blotting, and although HCC4006 GR3 cells clearly expressed AXL, they did not require the kinase for survival. In addition, while some reports show that aberrant Met activation via mutation or ligand activity secreted by stromal cells induces EMT and promotes invasion of carcinoma cells in many types of cancers, ${ }^{35-37}$ the GR cells characterized in this study did not depend on Met signaling for survival. This is in line with previous work by others showing that EGFR TKI-resistant EGFR-mutant cells with an EMT phenotype were uniformly not dependent on Met kinase. ${ }^{5-7}$ Collectively, these findings suggest that Met signaling does not have a key role in inducing or maintaining EMT of EGFR-mutant lung cancer cells.

In conclusion, two EGFR-mutant lung cancer cell lines have subsets of GR cells that have undergone EMT and can survive independent of EGFR activity. The presence of cells that are not EGFR addicted in EGFR-mutant lung cancer may account for the inability of EGFR TKIs to eliminate EGFRmutant lung cancers. It is likely that high levels of basal autophagy in GR cells facilitate the survival of these cells under hypoxic conditions in vitro. Moreover, some carcinoma cells contain autophagosomes even in untreated EGFRmutant lung cancer tissues in vivo, providing further evidence for the role of autophagy in these tissues. Considering these experimental findings and that cancer tissues usually have hypoxic regions, ${ }^{29,30}$ targeting autophagy could be a valuable new approach to treat these cancers. 


\section{ACKNOWLEDGEMENTS}

We thank Ms Kiyomi Hidano and Mr Takaaki Nikaido for their excellent electron microscopy. This work was supported in part by a Grant-in-Aid for Scientific Research (C) (24590440) from Japan Society for the Promotion of Science (to YS).

\section{DISCLOSURE/CONFLICT OF INTEREST}

The authors declare no conflict of interest.

1. Sharma SV, Bell DW, Settleman J, et al. Epidermal growth factor receptor mutations in lung cancer. Nat Rev Cancer 2007;7:169-181.

2. Pao W, Chmielecki J. Rational, biologically based treatment of EGFRmutant non-small-cell lung cancer. Nat Rev Cancer 2010;10:760-774.

3. Zhou W, Ercan D, Chen L, et al. Novel mutant-selective EGFR kinase inhibitors against EGFR T790M. Nature 2009;462:1070-1074.

4. Nakagawa T, Takeuchi S, Yamada T, et al. Combined therapy with mutant-selective EGFR inhibitor and Met kinase inhibitor for overcoming erlotinib resistance in EGFR-mutant lung cancer. Mol Cancer Ther 2012;11:2149-2157.

5. Yao Z, Fenoglio S, Gao DC, et al. TGF-beta IL-6 axis mediates selective and adaptive mechanisms of resistance to molecular targeted therapy in lung cancer. Proc Natl Acad Sci USA 2010;107:15535-15540.

6. Suda K, Tomizawa K, Fujii M, et al. Epithelial to mesenchymal transition in an epidermal growth factor receptor-mutant lung cancer cell line with acquired resistance to erlotinib. J Thorac Oncol 2011;6: 1152-1161.

7. Chung JH, Rho JK, $\mathrm{Xu} \mathrm{X}$, et al. Clinical and molecular evidences of epithelial to mesenchymal transition in acquired resistance to EGFRTKIs. Lung Cancer 2011;73:176-182.

8. Sequist LV, Waltman BA, Dias-Santagata D, et al. Genotypic and histological evolution of lung cancers acquiring resistance to EGFR inhibitors. Sci Transl Med 2011;3:75ra26.

9. Zhang $Z$, Lee $\mathrm{JC}$, Lin $\mathrm{L}$, et al. Activation of the $\mathrm{AXL}$ kinase causes resistance to EGFR-targeted therapy in lung cancer. Nat Genet 2012;44:852-860.

10. White E. Deconvoluting the context-dependent role for autophagy in cancer. Nat Rev Cancer 2012;12:401-410.

11. Kreuzaler P, Watson CJ. Killing a cancer: what are the alternatives? Nat Rev Cancer 2012;12:411-424.

12. Shen $\mathrm{S}$, Kepp $\mathrm{O}$, Michaud $\mathrm{M}$, et al. Association and dissociation of autophagy, apoptosis and necrosis by systematic chemical study. Oncogene 2011;30:4544-4556.

13. Guo JY, Chen HY, Mathew R, et al. Activated Ras requires autophagy to maintain oxidative metabolism and tumorigenesis. Genes Dev 2011;25:460-470.

14. Yang $\mathrm{S}$, Wang $\mathrm{X}$, Contino $\mathrm{G}$, et al. Pancreatic cancers require autophagy for tumor growth. Genes Dev 2011;25:717-729.

15. Zois $C E$, Giatromanolaki $A$, Sivridis $E$, et al. 'Autophagic flux' in normal mouse tissues: focus on endogenous LC3A processing. Autophagy 2011;7:1371-1378.

16. Bai $\mathrm{H}$, Inoue J, Kawano $\mathrm{T}$, et al. A transcriptional variant of the LC3A gene is involved in autophagy and frequently inactivated in human cancers. Oncogene 2012;31:4397-4408.

17. Sato K, Tsuchihara K, Fujii S, et al. Autophagy is activated in colorectal cancer cells and contributes to the tolerance to nutrient deprivation. Cancer Res 2007;67:9677-9684.

18. Ladoire S, Chaba K, Martins I, et al. Immunohistochemical detection of cytoplasmic LC3 puncta in human cancer specimens. Autophagy 2012;8:1175-1184.
19. Lee JG, Wu R. Combination erlotinib-cisplatin and Atg3-mediated autophagy in erlotinib resistant lung cancer. PLoS ONE 2012;7: e48532.

20. Li YY, Lam SK, Mak JC, et al. Erlotinib-induced autophagy in epidermal growth factor receptor mutated non-small cell lung cancer. Lung Cancer 2013;50169-5002:00224-00229 (in press).

21. Verdoodt B, Vogt $\mathrm{M}, \mathrm{Schmitz}$ I, et al. Salinomycin induces autophagy in colon and breast cancer cells with concomitant generation of reactive oxygen species. PLoS ONE 2012;7:e44132.

22. Matsukuma S, Yoshihara M, Kasai F, et al. Rapid and simple detection of hot spot point mutations of epidermal growth factor receptor BRAF, and NRAS in cancers using the loop-hybrid mobility shift assay. J Mol Diagn 2006;8:504-512.

23. Matsukuma S, Saito H, Yamada K, et al. Simple and precise detection of UGT1A1 polymorphisms with a modified loop-hybrid mobility shift assay using Cy5-labeled loop probes. Clin Chim Acta 2011;412: 1668-1672.

24. Matsukuma $\mathrm{S}$, Yoshihara $\mathrm{M}$, Suda $\mathrm{T}$, et al. Differential detection of KRAS mutations in codons 12 and 13 with a modified loop-hybrid (LH) mobility shift assay using an insert-type LH-generator. Clin Chim Acta 2011;412:1874-1878.

25. Sakuma Y, Yamazaki Y, Nakamura Y, et al. WZ4002, a third-generation EGFR inhibitor, can overcome anoikis resistance in EGFR-mutant lung adenocarcinomas more efficiently than Src inhibitors. Lab Invest 2012;92:371-383.

26. Sakuma $\mathrm{Y}$, Yamazaki $\mathrm{Y}$, Nakamura $\mathrm{Y}$, et al. NF- $\mathrm{kB}$ signaling is activated and confers resistance to apoptosis in three-dimensionally cultured EGFR-mutant lung adenocarcinoma cells. Biochem Biophys Res Commun 2012;423:667-671.

27. Yang MH, Wu MZ, Chiou SH, et al. Direct regulation of TWIST by HIF1alpha promotes metastasis. Nat Cell Biol 2008;10:295-305.

28. Sun S, Ning X, Zhang Y, et al. Hypoxia-inducible factor-1alpha induces Twist expression in tubular epithelial cells subjected to hypoxia, leading to epithelial-to-mesenchymal transition. Kidney Int 2009;75: 1278-1287.

29. Brown JM, Wilson WR. Exploiting tumour hypoxia in cancer treatment. Nat Rev Cancer 2004:4:437-447.

30. Denko NC. Hypoxia, HIF1 and glucose metabolism in the solid tumour. Nat Rev Cancer 2008;8:705-713.

31. Zhu W, Chen J, Cong X, et al. Hypoxia and serum deprivation-induced apoptosis in mesenchymal stem cells. Stem Cells 2006;24:416-425.

32. Corbin AS, Agarwal $A$, Loriaux $M$, et al. Human chronic myeloid leukemia stem cells are insensitive to imatinib despite inhibition of BCR-ABL activity. J Clin Invest 2011;121:396-409.

33. Bardsley MR, Horváth VJ, Asuzu DT, et al. Kit ${ }^{\text {low }}$ stem cells cause resistance to Kit/platelet-derived growth factor alpha inhibitors in murine gastrointestinal stromal tumors. Gastroenterology 2010;139: 942-952.

34. Bellodi C, Lidonnici MR, Hamilton A, et al. Targeting autophagy potentiates tyrosine kinase inhibitor-induced cell death in Philadelphia chromosome-positive cells, including primary CML stem cells. J Clin Invest 2009;119:1109-1123.

35. Boccaccio C, Comoglio PM. Invasive growth: a MET-driven genetic programme for cancer and stem cells. Nat Rev Cancer 2006;6: 637-645.

36. Gherardi E, Birchmeier W, Birchmeier C, et al. Targeting MET in cancer: rationale and progress. Nat Rev Cancer 2012;12:89-103.

37. Knight JF, Lesurf $R$, Zhao $H$, et al. Met synergizes with $p 53$ loss to induce mammary tumors that possess features of claudin-low breast cancer. Proc Natl Acad Sci USA 2013;110:E1301-E1310. 